\title{
Viscoelasticity of cellulose polymers and mucociliary transport on frog palates
}

\author{
Shun Y. Lin ${ }^{\mathrm{a}}$, Gordon L. Amidon ${ }^{\mathrm{a}}$, Normal D. Weiner ${ }^{\mathrm{a}}$ and Arthur H. Goldberg ${ }^{\mathrm{b}}$ \\ ${ }^{a}$ College of Pharmacy, The University of Michigan, Ann Arbor, MI 48109.1065 (USA) and ${ }^{b}$ Rugby-Darby Group Companies, \\ Inc., Rockville Centre, NY 11570 (USA)
}

(Received 23 March 1992)

(Modified version received 26 August 1992)

(Accepted 8 October 1992)

Key words: Cellulose polymer; Contour plot; Mixed solvent system; Moisture absorption; Viscoelasticity; Mucociliary transport

\begin{abstract}
Summary
The viscoelastic properties of model polymer, hydroxypropylmethylcellulose (HPMC), in ternary solvent mixtures and the variation of viscoelastic properties under dilution with water were investigated. HPMC was dispersed in mixtures of glycerol formal (GF) or ethyl alcohol, propylene glycol (PG), and water. Each polymeric sample was oscillated at different frequencies with fixed stress amplitude and the changes in elastic modulus, viscous modulus, and apparent viscosity were measured at $30^{\circ} \mathrm{C}$. The moisture absorption rate of HPMC in PG:GF solvent mixtures in a $94 \%$ relative humidified environment was also studied. A linear relationship between the elastic modulus or viscous modulus and polymer concentration was observed for the HPMC samples. The relative mucociliary transport rate tested on the non-depleted frog palate model revealed a curvilinear correlation with the loss tangent (a ratio of the viscous modulus to the elastic modulus) of the polymeric solution. Using GF as a solvent for HPMC resulted in a formation of a rigid gel with the highest elastic modulus and viscous modulus among solvents selected. A higher affinity for water was found for HPMC in the PG:GF 90:10 mixture compared with HPMC in the PG: GF 70:30 mixture. However, the elastic modulus and viscous modulus were much higher for HPMC in the PG: GF $70: 30$ mixture.
\end{abstract}

\section{Introduction}

In recent years, water soluble cellulose polymers have been used in a variety of pharmaceutical application (Peppas, 1987). The water soluble celluloses are ethers of cellulose with various degreees of substitution, both ionic and nonionic. The physical properties of these modified cellulose can be varied by the degree of polymeriza-

Correspondence to: G.L. Amidon, College of Pharmacy, The University of Michigan, Ann Arbor, MI 48109-1065, U.S.A. tion and the type of substitution. As linear polymers, cellulose derivatives undergo swelling in the aqueous medium until complete dispersion. The viscosity reaches a maximum in the swollen mass and then diminishes due to the progressive dilution of solubilized material. Also, due to entaglement and association of the polymer chains in aqueous solution, the apparent viscosity decreases with increased shearing rates. This phenomenon is generally more pronounced at higher shearing rate conditions and for higher molecular weight polymers (Bird et al., 1987).

The adhesive forces between polymers and natural tissue have received considerable atten- 
tion in drug delivery in recent years (Peppas et al., 1987; Leung et al., 1990). There have been reports that the adhesive strength increases as the molecular weight of the polymer increases up to 100,000 (Gurny et al., 1984; Hunt et al., 1987; Gurny and Peppas, 1990). According to the fracture theory of bioadhesion, the failure of an adhesive bond between two polymer surfaces is the result of disentanglement and/or cleavage of macromolecular chains (Ponchel et al., 1987). A critical length of the molecules is necessary to produce the interprenetrating layer and molecular entanglements between the bioadhesive and the substrate (Hunt et al., 1987). In the region of very low concentrations the interaction between polymer molecules is not significant (Bird et al., 1987). At these concentrations, the changes of physical properties is in direct proportion to the polymer concentration. When the polymer concentration increases, the increment in viscosity over that of the solvent increases at a faster rate which means the intermolecular entanglements dominate the dynamics of polymeric solutions. The coiled molecules become solvent-poor and the available chain length for interfacial penetration decreases.

For an adhesive formulation designed for delivering drugs to the respiratory tract, the adhesive is subjected to oscillatory deformation created by cilia movement. Understanding the viscoelastic properties of adhesives under oscillation may provide some insightful information for enhancing the residence time of the formulation in the respiratory tract. The objective of this investigation is to evaluate (a) the variation of viscoelastic properties of HPMC in mixed solvent systems and the relationships between viscoelasticity and mucociliary transport; and (b) the effects of water on the elastic modulus and viscous modulus of HPMC in PG: GF solvent systems.

\section{Materials and Methods}

\section{Materials}

The polymeric materials tested were hydroxypropylmethylcellulose (HPMC grade E10M, K15M, and K100M, Dow Chemical, Midland, MI) and polyethylene oxide (Polyox grades $600 \mathrm{~K}, 2 \mathrm{M}$, and $5 \mathrm{M}$, Union Carbide, Danbury, CT). These polymers were used without any purification. The number average molecular weight $\left(M_{\mathrm{n}}\right)$ is approx. 85000 for HPMC-E10M, 100000 for HPMC$\mathrm{K} 15 \mathrm{M}$, and 150000 for HPMC-K100M. The apparent viscosity is $4900 \mathrm{cps}$ for $5 \%$ Polyox- $600 \mathrm{~K}$, $3450 \mathrm{cps}$ for $2 \%$ Polyox-2M, and $6200 \mathrm{cps}$ for $1 \%$ Polyox-5M. Several non-aqueous solvents were selected as potential solvating agents for HPMC, including propylene glycol, polyethylene glycol 400 , propylene carbonate, glycerol formal, methyl pyrrolidione, $N, N$-dimethyl acetamide, ethyl lactate, 1,2-o-isopropylidene-rac-glycerol (the solvents mentioned above were purchased from Sigma, St. Louis, MO), and ethyl alcohol (200 proof, Aaper Alcohol and Chemical, Shelbyville, $\mathrm{KY}$ ).

\section{Methods}

\section{Measurement of viscoelastic properties}

There are two main techniques for evaluating rheologic properties of polymeric solutions: long time scale (transient test) and short time scale (dynamic test). Both methods allow one to measure the elasticity (elastic modulus, $G^{\prime}$ ) and viscosity (viscous modulus, $G^{\prime \prime}$ ) of samples. In this study, a Rheo-Tech ViscoElastic Rheometer (Contraves Co., Cincinnati, $\mathrm{OH}$ ) was used to measure the changes of viscoelasticity under oscillation, a short time scale measurement. The oscillatory deformation of polymeric samples was tested using either a 'cone and plate' or a 'coaxial cylinder' measuring system. The cone and plate system has $1^{\circ}$ in angle with $5.0 \mathrm{~cm}$ in diameter and the gap between the cone and the plate is $0.05 \mathrm{~mm}$. In the coaxial cylinder system, the cup has $14.1 \mathrm{~mm}$ in diameter and the bob has $13 \mathrm{~mm}$ in diameter with $19.5 \mathrm{~mm}$ in length. Different amounts of HPMC-E10M were dissolved in water to prepare polymeric samples at $1.5,2.0,2.5,3.0$, $3.5,4.0$, and $6 \% \mathrm{w} / \mathrm{w}$ samples. However, only $4 \%$ $\mathrm{w} / \mathrm{w}$ of Polyox samples in water were studied. The polymeric samples were mixed vigorously for 2 min and stored overnight at room temperature. Samples were mixed again on the second day and were centrifuged at $2500 \mathrm{rpm}$ for $20 \mathrm{~min}$ at room temperature with a Beckman J2-21M Centrifuge 
(Palo Alto, CA). After removing the air bubbles, samples were not disturbed until testing for the viscoelastic properties. Some samples were divided into two portions, one for the mucociliary transport study on frog palate and another for the viscoelasticity measurements.

For the viscoelasticity measurement, sufficient amount of sample was loaded and the rotating rod (cone or bob) was lowered slowly to the position to minimize the shear deformation on testing sample. A thin layer of light paraffin oil was applied to prevent the evaporation of solvents and the temperature of measuring system was maintained at $30 \pm 0.3^{\circ} \mathrm{C}$. The apparent viscosity of samples was also measured using the equilibrium flow curve testing system. Samples were first amplitude scanned at frequency $1.0 \mathrm{~Hz}$ to identify the linear viscoelastic region before measuring the elastic modulus and viscous modulus, a region where the elastic modulus and the viscous modulus are independent of stress amplitude (driving torque). A stress amplitude was selected from this region for frequency scan with a strain amplitude of sufficient magnitude to ensure a good signal-to-noise ratio. Usually, the strain amplitude was maintained between $1 \times$ $10^{-3}$ and $1 \times 10^{-2} \mathrm{mN} \mathrm{m}$ during the oscillation test. The oscillatory frequencies were operated from 0.01 to $2.5 \mathrm{~Hz}$ and the changes of elastic modulus and viscous modulus were recorded. The apparent viscosity at coordinated shear rate was obtained from the linear relationship between the logarithm of viscosity and shear rate.

In oscillatory shear, the response of viscoelastic materials to a small amplitude oscillatory shear is treated as follows (Ferry, 1980; Barnes et al., 1989): The absolute complex modulus $\left|G^{*}\right|=$ $\left(G^{\prime 2}+G^{\prime \prime 2}\right)^{1 / 2}$ with the elastic modulus $\left(G^{\prime}\right)=$ $\left(\sigma_{\mathrm{o}} / \gamma_{\mathrm{o}}\right) \cos \delta$ and the viscous modulus $\left(G^{\prime \prime}\right)=$ $\left(\sigma_{o} / \gamma_{o}\right) \sin \delta$, where $\sigma_{o}$ is the initial stress amplitude, $\gamma_{o}$ is the initial strain amplitude, and $\delta$ is the phase angle between stress and strain. The absolute complex viscosity $\left|\eta^{*}\right|=\left(G^{2}+\right.$ $\left.G^{\prime \prime 2}\right)^{1 / 2} / 2 \pi \omega$ where $\omega$ is the radial velocity.

\section{Viscoelasticity of mixed solvent systems}

Several non-aqueous solvents were used to dissolve 4 or $6 \% \mathrm{w} / \mathrm{w}$ HPMC and the behavior of sample's viscoelasticity was studied. However, only those exhibiting gel formation were tested for their viscoelastic properties. A surface tensiometer (Rosano, Laboratory Products, Inc., Boston, MA) was used to determine the surface tension of pure solvent at room temperature. Among these non-aqueous solvents, glycerol formal (GF) and ethyl alcohol (ALC) representing two extremes, were selected for further study. 31 HPMC samples were prepared to construct the contour diagrams of elastic modulus and viscous modulus in either PG-GF- $\mathrm{H}_{2} \mathrm{O}$ or PG-ALC- $\mathrm{H}_{2} \mathrm{O}$ system. The solvent compositions in each sample were predetermined on a triangular plot. The elastic modulus and viscous modulus measured at $1 \mathrm{~Hz}$ were analyzed using Scheffe-type polynomial model with the help of a statistical program (SAS). A computer program of Sygraph (Systat Intelligent Software) was employed to draw the ternary diagram contour maps.

\section{Measurement of moisture uptake}

$6 \% \mathrm{w} / \mathrm{w}$ HPMC-E10M was dispersed in a mixture of PG and GF as the stock solutions in this study. $10 \mathrm{~g}$ of each stock solution containing either $90: 10$ or $70: 30 \mathrm{PG}$ : GF was transferred to a $20 \mathrm{ml}$ liquid scintillation vial. The bulk density of stock solutions was determined by weighing sample in triplicate in a $10 \mathrm{ml}$ calibrated volumetric flask. The changes of viscoelastic properties after diluting with water were measured by adding different amounts of deionized water in the vial with the final degree of dilution with water varied from 0 to $235 \%$ of its initial weight. Some HPMC-E10M samples were prepared by weighing exact volume at $1,2,3,4 \mathrm{ml}$ for each mixed solvent system and transferred to glass petri dishes with $50 \mathrm{~mm}$ in diameter and $15 \mathrm{~mm}$ in height. Samples were studied in triplicate in a laboratory-designed humidity chamber. This humidity chamber composed a temperature controlled water bath, a 101 container with opened top (24.5 $\mathrm{cm}$ in diameter and $25.5 \mathrm{~cm}$ in height), a desiccator plate, a temperature and relative humidity indicator, a small custom-designed electrical fan (speed of rotation, $600 \mathrm{rpm}$ ), and an insulated board. Samples were placed on top of the desiccator plate which was $4 \mathrm{~cm}$ above the bottom of 
container. A small amount of deionized water was filled into the container $(<500 \mathrm{ml})$ with temperature kept at $29 \pm 1^{\circ} \mathrm{C}$ and covered with the insulated board. The fan was secured on the insulated board with generator (model CA, Hurst, Princeton, IN) on the opposite side of container to prevent heat transfer and the relative humidity was maintained at $94 \pm 1 \%$. The changes of weight in each polymeric sample after $0,10,20$, $30,40,50,80,100$, and $120 \mathrm{~min}$ incubation in the humidity chamber were recorded.

\section{Mucociliary transport on frog palate}

Bullfrogs (Rana catesbiana), 5 inches or larger, were killed by decapitation. The lower jaw was removed with scissors and the upper palate was washed with frog Ringer solution $(90 \mathrm{mM} \mathrm{NaCl}, 3$ $\mathrm{mM} \mathrm{KCl}, 2 \mathrm{mM}$ Call $\cdot 2 \mathrm{H}_{2} \mathrm{O}$ and $15 \mathrm{mM}$ $\mathrm{NaHCO}_{3}, \mathrm{pH} 8$ ) and transferred to an $8 \times 4 \mathrm{~cm}^{2}$ glass petri dish covered with a $16.5 \times 16.5 \mathrm{~cm}^{2}$ glass plate with temperature kept within $25-28^{\circ} \mathrm{C}$. The mucociliary transport of each polymeric sample was measured using an eyepiece micrometer set in a stereo microscope at $\times 20$ magnification (Stereo 2, Cambridge Instruments) with activated charcoal powder (100 mesh, $150 \mu \mathrm{m})$ as the indicator. The surface of the palate was washed and wiped clear after each comparison of charcoal powder vs sample to remove any built-up of mucus. Whenever the baseline clearance rate of the charcoal powder was less than $10 \%$ of the initial clearance rate measured after excision, the palate was rested in a plastic container filled with cold frog ringer solution and stored for $45 \mathrm{~min}$ at $4^{\circ} \mathrm{C}$ before reuse.

\section{Results and Discussion}

Cellulose polymer is one of the popular polymers used today as a bioadhesive to prolong and control drug release. For a specific dose of drug, varying the polymer concentration is perhaps the most efficient way to adjust the release characteristics for a specific criterion. The effects of polymer concentration on the viscoelastic properties of HPMC-E10M solution are shown in Fig. 1. A linear relationship is observed between the dy-

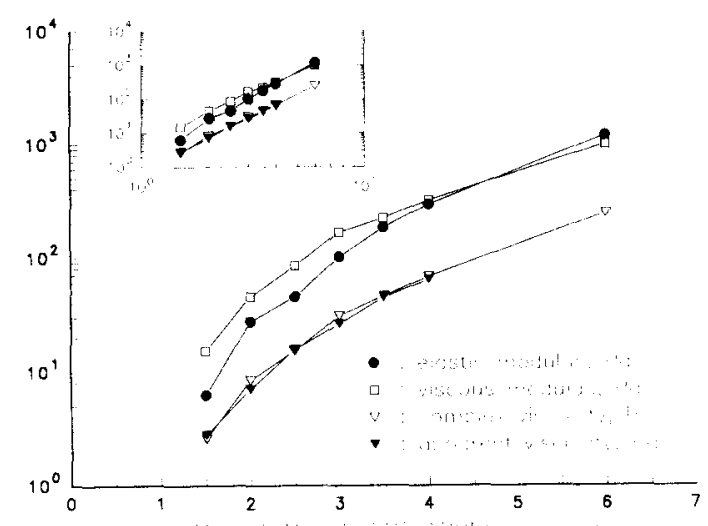

Fig. 1. The rheology of hydroxypropylmethylcellulose aqueous solutions (HPMC-E10M) with varying concentrations (measured at $1 \mathrm{~Hz}$ ).

namic moduli (elastic modulus and viscous modulus) and the concentration of E10M on a double$\log$ plot (Fig. 1). After increasing polymer concentration up to $6.0 \% \mathrm{w} / \mathrm{w}$, the elastic modulus of E10M aqueous solution measured at $1 \mathrm{~Hz}$ became the major component in the viscoelastic profiles. Although the loss tangent $\left(G^{\prime \prime} / G^{\prime}\right)$ equaled unity after increasing the $E 10 \mathrm{M}$ concentration to $5.5 \% \mathrm{w} / \mathrm{w}$, this tangent value is vary depended on the force (stress) and shearing rate (oscillatory frequency) applied. Because at higher shearing rates with constant stress the loss tangent will equal one at lower concentration. Since the effective shear rate of the nasal mucus layer is around $0.5-3 \mathrm{~Hz}$ induced by ciliary movement (Majima et al., 1990), data shown in Fig. 1 can be used to estimate the rheologic changes in E10M solutions as a function of concentration under ciliary movement (without mucus layer). It is desirable to predict the shearing rate dependent viscosity (apparent viscosity, $\eta$ ) from the linear viscoelastic measurement. The relations between linear viscoelastic properties and viscometric functions have been explored in the field of polymeric fluids. The Cox-Merz rule has been suggested as a way of obtaining an improved relationship between the linear viscoelastic properties and the viscosity (Ferry, 1980; Bird et al., 1987; Barnes et al., 1989). This empiricism predicts that the magnitude of the complex viscosity 
is equal to the viscosity at corresponding values of radial velocity $(\omega)$ and shearing rate $(\gamma)$ :

$$
\begin{aligned}
\eta(\gamma)=\left|\eta^{*}(\omega)\right| & \left.\right|_{\omega=\gamma} \\
& =\left.\eta^{\prime}(\omega)\left[1+\left(\eta^{\prime \prime} / \eta^{\prime}\right)^{2}\right]^{0.5}\right|_{\omega=\gamma}
\end{aligned}
$$

The agreements between $\left|\eta^{*}\right|$ and $\eta$ at a shearing rate of $6.3 \mathrm{~s}^{-1}$ shown in Fig. 1 are within experimental error for the linear polymeric samples. The influences of solvent on the viscoelastic properties of $6 \% \mathrm{E} 10 \mathrm{M}$ are shown in Table 1. The solvents tested covered a wide range of solubility parameters, dielectric constants, and surface tensions. There were three distinguished behaviors of viscoelastic properties of HPMCs in these solvents: (1) HPMC did not dissolve in propylene glycol (PG) or ethyl alcohol (ALC); (2) HPMC formed a gel in water, GF, or methyl pyrrolidione (PYR); and (3) the viscoelastic properties of HPMC in dimethyl acetamide (DMA), ethyl lactate (LAC), or solketal (SOL) are very shear stress dependent. HPMC formed a rigid gel structure in glycerol formal (GF) with highest dynamic moduli among solvents tested. Usually, the behavior of high molecular weight polymers examined under the stress deformation at low frequency, a tendency of $G^{\prime}$ crossover with $G^{\prime \prime}$ will be observed. An intercept between the elastic modulus $\left(G^{\prime}\right)$ and the viscous modulus $\left(G^{\prime \prime}\right)$ mea- sured at $1 \mathrm{~Hz}$ was found for $6 \% \mathrm{E} 10 \mathrm{M}$ in water or GF. The loss tangent of E10M in GF decreased as the oscillatory frequency increased. In fact, for diluted polymeric solution the loss tangent is very high because both solvent and polymer contribute to $G^{\prime \prime}$, but only the polymer contributes to $G^{\prime}$. At low frequencies, the loss tangent is large for all uncross-linked polymers and becomes inversely proportional to the frequency. The HPMC E type polymer (HPMC 2910) has a surface tension between 44 and $50 \mathrm{mN} / \mathrm{m}(1 \%$ solution in water) at room temperature and has a solubility parameter closed to 24 (Doelker, 1990). It is difficult to predict whether a solvent is a 'good solvent' for HPMC-E using only one parameter such as the solubility parameter, the dielectric constant, or the surface tension.

For a ternary solvents mixture containing one or more nonsolvents, eventually there will be a critical boundary (critical composition) where the polymer no longer is miscible with the solvent mixture. This boundary represents the minimum nonsolvent-solvent ratio where phase separation occurs at a given temperature. Although the polymer concentration in each ternary solvent system is kept constant, it can also be viewed as a variable in terms of polymer concentration in the soluble vehicle(s). Figs 2 and 3 show the contour variation of elastic modulus and viscous modulus of 4 or $6 \% \mathrm{E} 10 \mathrm{M}$ in one nonsolvent (PG-GF$\mathrm{H}_{2} \mathrm{O}$ ) system or in two non-solvents (PG-ALC-

TABLE 1

The rheology of $6 \% \mathrm{w} / \mathrm{w}$ hydroxypropylmethylcellulose (E1OM) in non-aqueous solvents

\begin{tabular}{llllllll}
\hline Solvent & Phase & $\begin{array}{l}\text { Elastic } \\
\text { modulus }\end{array}$ & $\begin{array}{l}\text { Complex } \\
\text { viscosity }\end{array}$ & $\begin{array}{l}\text { Loss } \\
\text { tangent }\end{array}$ & $\begin{array}{l}\text { Solubility } \\
\text { parameter a }\end{array}$ & $\begin{array}{l}\text { Dielectric } \\
\text { constant } \begin{array}{l}\text { a } \\
\text { tension } \\
\text { (dyne /cm) }\end{array}\end{array}$ \\
\hline Water & G & 1010 & 207 & 0.81 & 23.4 & 78.5 & 79.8 \\
ALC & S & ND & ND & ND & 12.7 & 24.3 & 25.0 \\
PG & S & ND & ND & ND & 12.6 & 32.0 & 41.4 \\
PYR & G & 911 & 185 & 0.79 & 11.3 & 32.2 & 45.5 \\
DMA & G & 718 & 137 & 0.66 & 10.8 & 37.8 & 40.1 \\
LAC & G & 641 & 135 & 0.87 & 10.0 & ND & 32.7 \\
SOL & G & 234 & 60 & 1.25 & 10.3 & 17.6 & 36.9 \\
GF & G & 4500 & 761 & 0.36 & 8.2 & ND & 51.3 \\
\hline
\end{tabular}

${ }^{a}$ From: Chien (1984), Rubino and Yalkowsky (1987) and Rajagopalan et al. (1988). G, gelled; S, separation; ND, not determined; ALC, absolute ethyl alcohol; PG, propylene glycol; PYR, methyl pyrrolidione; DMA, $N, N$-dimethylacetamide; LAC, ethyl lactate; SOL, 1,2-o-isopropylidene-rac-glycerol; GF, glycerol formal. 

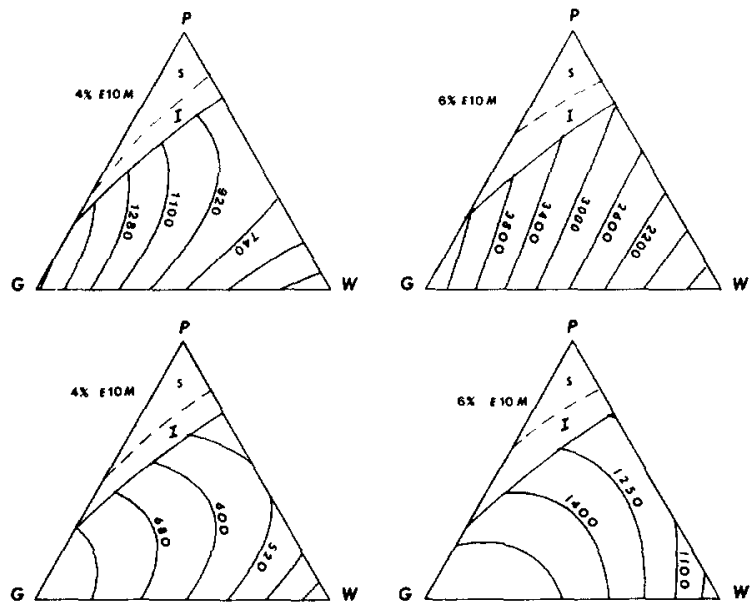

Fig. 2. Contour diagram of 4 and 6\% HPMC-E10M in propylene glycol (P), glycerol formal (G), and water (W) mixtures oscillated at $1 \mathrm{~Hz}$. (Top) Elastic modulus (Pa); (bottom) viscous modulus $(\mathrm{Pa})$; $(\mathrm{S})$ phase separation; (I) intermediate region.

$\left.\mathrm{H}_{2} \mathrm{O}\right)$ system. The regression correlation coefficient was higher than 0.99 in all triangular contour plots. By increasing polymer concentration from 4 to $6 \%, G^{\prime}$ increased significantly relative to $G^{\prime \prime}$. However, the shape and position of boundaries varied little as the polymer concentration changed. In the PG-GF- $\mathrm{H}_{2} \mathrm{O}$ system, the
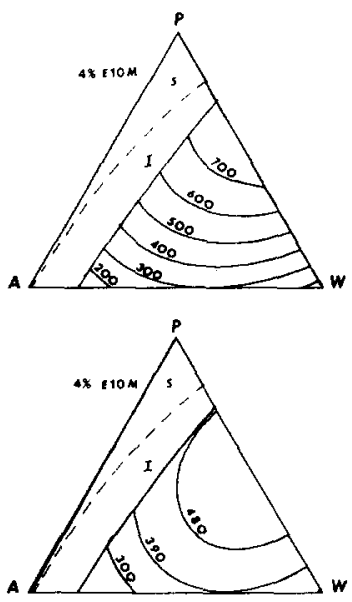
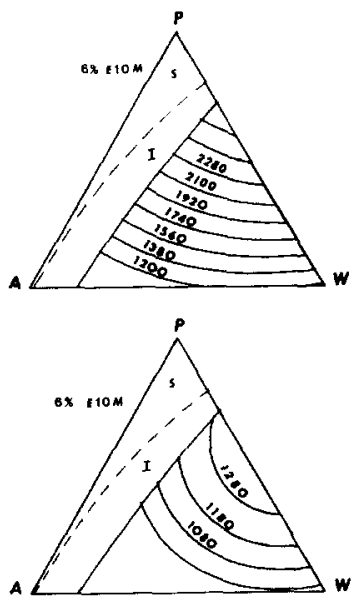

Fig. 3. Contour diagram of 4 and $6 \%$ HPMC-E10M in propylene glycol (P), ethyl alcohol (A), and water (W) mixtures oscillated at $1 \mathrm{~Hz}$. (Top) Elastic modulus (Pa); (bottom) viscous modulus $(\mathrm{Pa})$; (S) phase separation; (I) intermediate region.
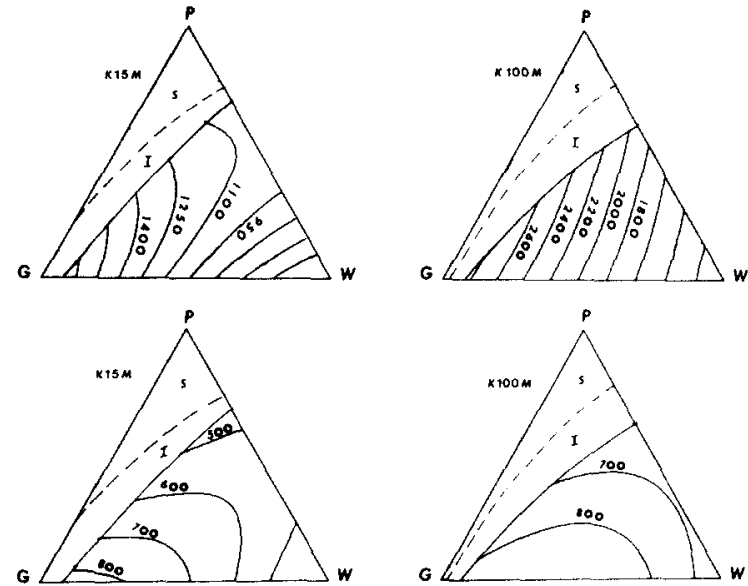

Fig. 4. Contour diagram of $4 \%$ HPMC-K15M and K100M in propylene glycol (P), glycerol formal (G), and water (W) mixtures oscillated at $1 \mathrm{~Hz}$. (Top) Elastic modulus ( $\mathrm{Pa}$ ); (bottom) viscous modulus $(\mathrm{Pa})$; (S) phase separation; (I) intermediate region.

highest $G^{\prime}$ and $G^{\prime \prime}$ were around $100 \% \mathrm{GF}$ and decreasing significantly when the percent of GF was reduced. For HPMC samples located in the intermediate phase (I region), between the separation region (S region) and gelled region (behind the solid boundary line), the loss tangent was very dependent on the stress applied. The E10M dissolved in the PG-GF binary solvent system when the amount of $P G$ was less than $60 \%$, in the PG- $\mathrm{H}_{2} \mathrm{O}$ system with PG less than $75 \%$, and in the $75: 25$ ALC- $\mathrm{H}_{2} \mathrm{O}$ system. This $75: 25$ ratio is close to the number reported for dissolving HPMC polymer type 2910 in ALC- $\mathrm{H}_{2} \mathrm{O}$ mixture (Doelker, 1990). However, the contours of $G^{\prime}$ and $G^{\prime \prime}$ in the PG-ALC- $\mathrm{H}_{2} \mathrm{O}$ system shift toward the PG- $\mathrm{H}_{2} \mathrm{O}$ line and center between $75: 25$ and $50: 50$ of $\mathrm{PG}: \mathrm{H}_{2} \mathrm{O}$ (Fig. 3).

Figs 4 and 5 show the viscoelastic properties of polymers HPMC-K (HPMC 2208). Because HPMC 2208 has a solubility parameter near 27 and has a surface tension between 50 and 56 $\mathrm{mN} / \mathrm{m}$ (Doelker, 1990), it is expected that GF will be a poor solvent for HPMC 2208. The solid boundary line moved toward $100 \%$ water, but the highest dynamic moduli still directed to $100 \%$ GF. Although the intrinsic viscosity and degree of side chain modification are different, the contour diagram of $4 \% \mathrm{~K} 15 \mathrm{M}$ did not show a significant 

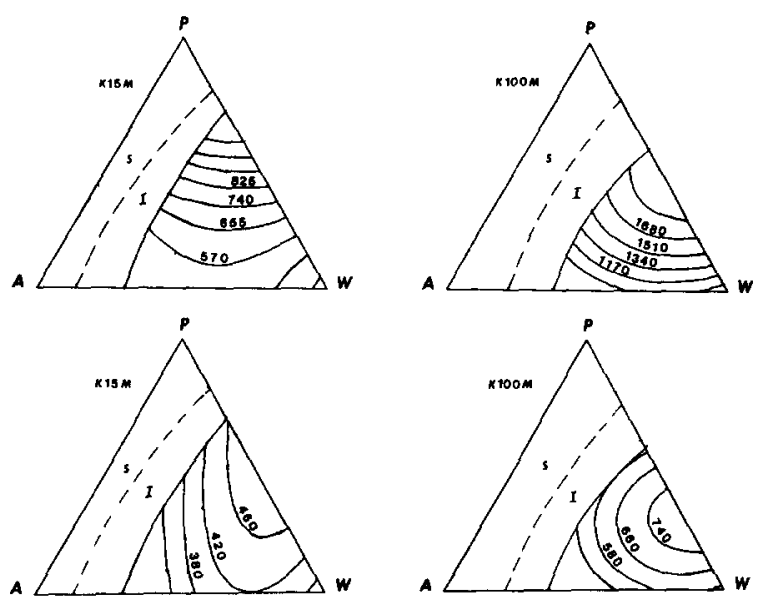

Fig. 5. Contour diagram of 4\% HPMC-K15M and K100M in propylene glycol $(\mathrm{P})$, ethyl alcohol $(\mathrm{A})$, and water $(\mathrm{W})$ mixtures oscillated at $1 \mathrm{~Hz}$. (Top) Elastic modulus (Pa); (bottom) viscous modulus $(\mathrm{Pa})$; (S) phase separation; (I) intermediate region.

difference compared with $4 \%$ E10M. The number average molecular weight $\left(M_{\mathrm{n}}\right)$ of HPMC is approx. 155000 for $\mathrm{K} 100 \mathrm{M}, 100000$ for $\mathrm{K} 15 \mathrm{M}$, and 85000 for E10M. Increasing the molecular weight of HPMC results in a more elastic behavior in the polymer viscoelastic profiles (Figs 4 and 5). It is reasonable to see that $4 \% \mathrm{~K} 100 \mathrm{M}$ is less soluble in non-aqueous solvents than $\mathrm{K} 15 \mathrm{M}$ and possesses higher dynamic moduli after dissolution (Figs 4 and 5). This is due to the greater chain length resulting in more entanglement of polymer molecules. The variation of viscoelastic profiles of

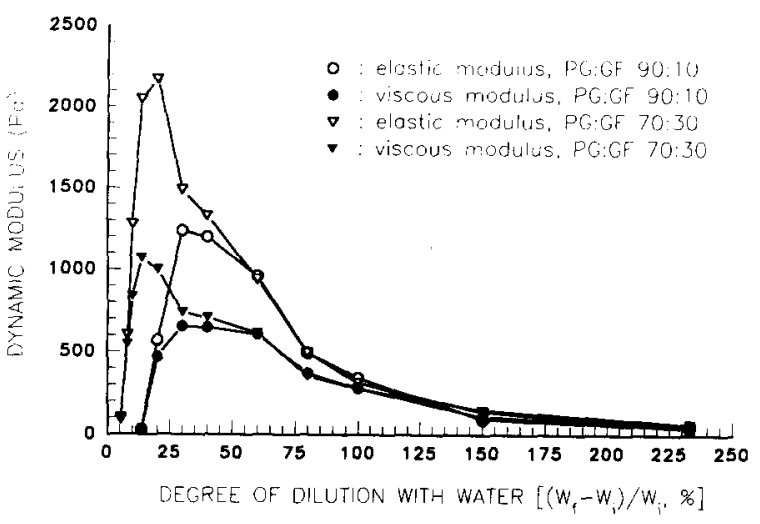

Fig. 6. Effect of water on the dynamic moduli of $6 \%$ HPMCE10M in mixed PG: GF systems. $W_{\mathrm{f}}$, final weight; $W_{\mathrm{i}}$, initial weight before adding water.

E10M to $\mathrm{K} 15 \mathrm{M}$ were more pronounced in the PG-ALC- $\mathrm{H}_{2} \mathrm{O}$ system than in the PG-GF- $\mathrm{H}_{2} \mathrm{O}$ system.

When a non-aqueous polymeric sample contacts water, both the composition of solvent and the dynamic mechanical properties of polymeric solution are altered. The measured densities for 6\% E10M in the PG:GF $90: 10$ and PG:GF $70: 30$ systems were $1.062 \pm 0.002$ and $1.089 \pm$ $0.003 \mathrm{~g} / \mathrm{ml}$, respectively. By increasing the water content in the preparations, both $G^{\prime}$ and $G^{\prime \prime}$ increase dramatically to a maximum and then decrease (Fig. 6). The decrease of $G^{\prime \prime}$ was much slower compared with $G^{\prime}$ and the amount of water required to form a gel structure was higher for the PG:GF 90:10 system than the PG:GF
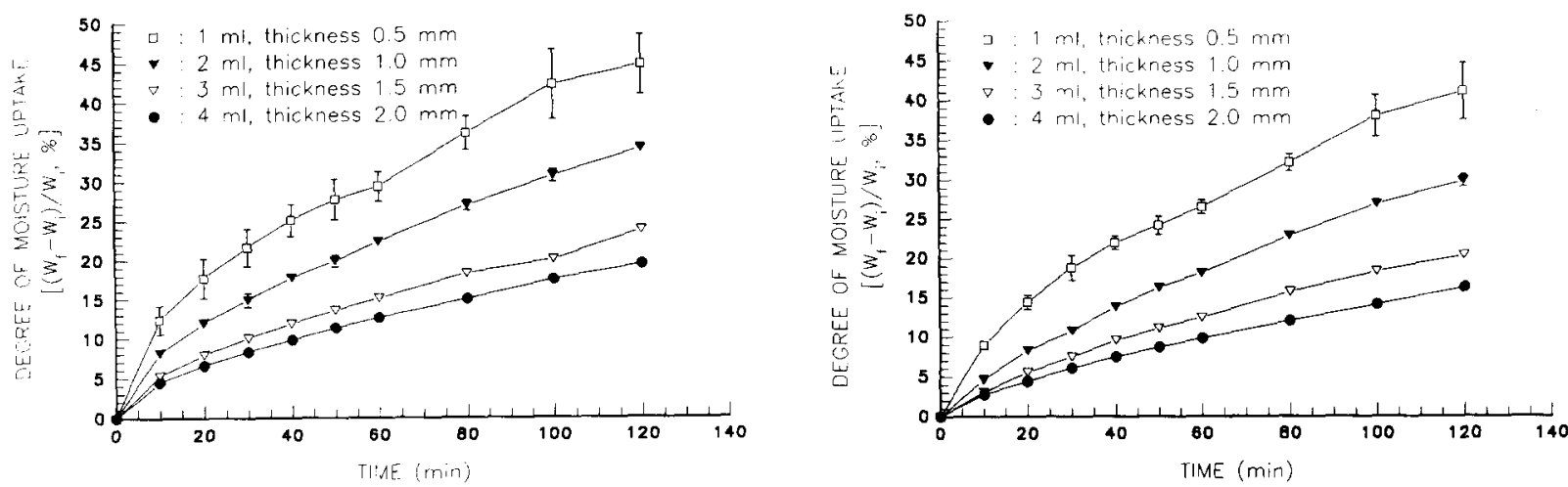

Fig. 7. (a) Effect of thickness on the moisture uptake of $6 \%$ HPMC-E10M in the PG: GF $90: 10$ system. $W_{\mathrm{f}}$, final weight; $W_{\mathrm{i}}$, initial weight before adding water. (b) Effect of thickness on the moisture uptake of $6 \%$ HPMC-E10M in the PG:GF $70: 30$ system. $W_{\mathrm{f}}$, final weight; $W_{i}$, initial weight before adding water. 

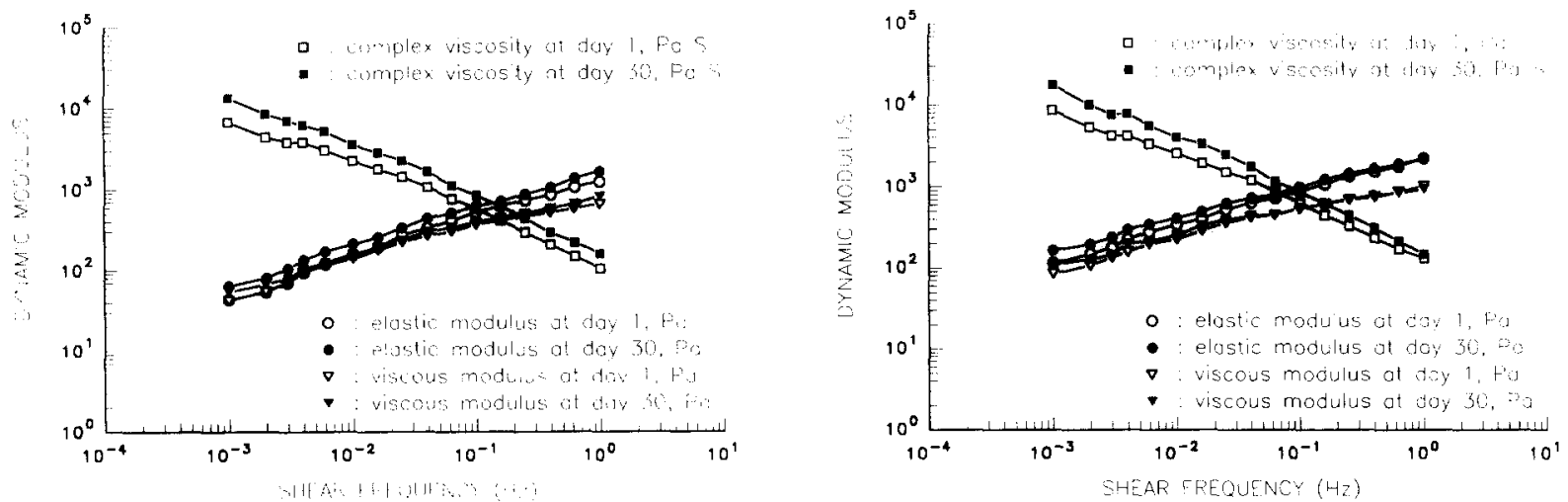

Fig. 8. (a) The stability of 6\% HPMC-E10M in the PG:GF 90:10 system after adding 30\% w/w water. (b) The stability of $6 \%$ HPMC-E10M in the PG : GF $70: 30$ system after adding $20 \% \mathrm{w} / \mathrm{w}$ water.

$70: 30$ system. Although $G^{\prime}$ and the $G^{\prime \prime}$ were higher initially in the PG: GF $70: 30$ system, the viscoelastic profiles of both systems were almost the same after diluting with water to $100 \%$ of their initial weights.

The ability of E10M samples in mixed nonaqueous solvents to absorb moisture from a humidified chamber is shown in Fig. 7a and b. The system with PG:GF 90:10 absorbed moisture faster than with PG: GF 70:30. This could be an advantage for the PG: GF $90: 10$ system to acquire the same initial dynamic moduli to form a gel structure as the PG:GF $70: 30$ system. The logarithm of moisture uptake rate for the PG: GF $70: 30$ polymeric system was inversely proportional to the square root of thickness and to the cubic root of thickness for the PG:GF 90:10 polymeric system. Fig. 8a and b shows the stability of HPMC in PG: GF $90: 10$ system with $30 \%$ $\mathrm{w} / \mathrm{w}$ water and in the PG: GF $70: 30$ system with

TABLE 2

The relative mucociliary transport of polymeric aqueous solutions on frog palates

\begin{tabular}{|c|c|c|c|c|c|c|}
\hline Polymer & $\begin{array}{l}\text { Elastic } \\
\text { modulus }\end{array}$ & $\begin{array}{l}\text { Viscous } \\
\text { modulus }\end{array}$ & $\begin{array}{l}\text { Complex } \\
\text { viscosity }\end{array}$ & $\begin{array}{l}\text { Apparent } \\
\text { viscosity }\end{array}$ & $\begin{array}{l}\text { Loss } \\
\text { tangent }\end{array}$ & $\begin{array}{l}\text { Relative } \\
\text { transport }\end{array}$ \\
\hline \multicolumn{7}{|c|}{ Hydroxypropylmethylcellulose (Methocel) } \\
\hline $2 \% \mathrm{E}-10 \mathrm{M}$ & $12.2 \mathrm{~Pa}$ & $37.9 \mathrm{~Pa}$ & $6.4 \mathrm{~Pa} \mathrm{~S}$ & $6.8 \mathrm{~Pa} \mathrm{~S}$ & 3.10 & $0.30 \pm 0.22$ \\
\hline $4 \% \mathrm{E}-10 \mathrm{M}$ & 214.0 & 248.0 & 52.1 & 51.6 & 1.16 & $0.15 \pm 0.01$ \\
\hline \multicolumn{7}{|c|}{ Polyethylene oxide (Polyox) } \\
\hline $4 \% 600 \mathrm{~K}$ & 2.9 & 9.3 & 1.6 & 1.0 & 3.21 & $0.28 \pm 0.05$ \\
\hline $4 \% 2 \mathrm{M}$ & 60.6 & 52.2 & 12.7 & 13.5 & 0.86 & $0.05 \pm 0.02$ \\
\hline $4 \% 5 \mathrm{M}$ & 246.0 & 81.1 & 41.2 & 32.2 & 0.33 & $0.08 \pm 0.01$ \\
\hline \multicolumn{7}{|c|}{ Na-carboxymethylcellulose (cellulose gum) } \\
\hline $2 \% 7 \mathrm{HF}$ & 39.1 & 48.3 & 9.9 & 13.4 & 1.24 & $0.26 \pm 0.07$ \\
\hline $4 \% 7 \mathrm{HF}$ & 614.0 & 306.0 & 109.0 & 97.9 & 0.50 & $0.04 \pm 0.02$ \\
\hline \multicolumn{7}{|c|}{ Na-alginate (Kelcosol) } \\
\hline $2 \%$ & 106.0 & 102.0 & 23.3 & 17.3 & 0.96 & $0.09 \pm 0.04$ \\
\hline $4 \%$ & 954.0 & 414.0 & 165.0 & 108.4 & 0.43 & $0.004 \pm 0.002$ \\
\hline \multicolumn{7}{|c|}{ Polyacrylic acid (Carbopol) } \\
\hline $1 \% 934 \mathrm{P}$ & 1440.0 & 66.3 & 226.0 & 60.2 & 0.05 & $0.36 \pm 0.17$ \\
\hline $2 \% 934 \mathrm{P}$ & 1840.0 & 86.5 & 293.0 & 99.2 & 0.05 & $0.37 \pm 0.11$ \\
\hline
\end{tabular}


$20 \% \mathrm{w} / \mathrm{w}$ water. Both figures show a clear intercept between $G^{\prime}$ and $G^{\prime \prime}$ lines for samples tested on the first day. However, the differences between elastic modulus and viscous modulus seemed to expand at lower frequencies after 30 days storage.

The mucociliary movement in the respiratory tract serves as a primary defense mechanism against inhaled materials under physiological conditions. A thin layer periciliary fluid exists between the mucus and epithelium where the cilia beat back (recovery stroke) and forth (effective stroke). The mucus in the mammalian respiratory tract acts only as a coupling agent for cilia to transport airborne and other particles. Within a moderate range of deviation from 'normal', the ciliary beat frequency (Phillips et al., 1990) and the mucus viscoelasticity (Gelman and Meyer, 1979) are direct factors which influence the mucociliary transport rate. Polymeric samples with different viscoelastic properties are cleared at different transport rates in the nasal cavity (Hardy et al., 1985; Illum, 1986; Gonda et al., 1990). Table 2 shows the results of mucociliary transport rate of polymeric solutions tested on non-depleted frog palates. It is clearly shown in this investigation that the mucociliary transport rates are reduced at different fashions after applying polymeric preparations with different viscoelastic properties. Among the viscoelastic properties tested, the loss tangent provided a better curvilinear correlation with the relative mucociliary transport rate. Information such as the viscoelastic properties of polymeric (bioadhesive) preparation under oscillation could provide some insight on the behaviors of the formulation in the respiratory tract.

\section{References}

Barnes, H.A., Hutton, J.F, and Walters, K. An Introduction to Rheology, Elsevier, Amsterdam, 1989, pp. 37-114.

Bird, R.B., Curtiss, C.F., Armstrong, R.C. and Hassager, O., Dynamics of Polymeric Liquids, 2nd Edn, Vol. I: Fluid Mechanics, Wiley, New York, 1987, pp. 55-168.

Chien, Y.W., Solubilization of metronidazole by water-miscible multi-cosolvents and water-soluble vitamins. J. Parenter. Sci. Technol., 38 (1984) 32-36.
Doelker, E., Swelling behavior of water-soluble cellulose derivatives. In Brannon-Peppas, L. and Harland, R.S. (Eds), Absorbent Polymer Technology, Amsterdam, 1990, pp. 125-146.

Ferry, J.D., Viscoelastic Properties of Polymers, 3rd Edn, Wiley, New York, Inc., 1980, pp. 33-129.

Gelman, R.A. and Meyer, F.A., Mucociliary transference rate and mucus viscoelasticity. Dependence on dynamic storage and loss modulus. Am. Rev. Respir. Dis. 120 (1979) 553557.

Gonda, I. and Gipps, E., Model of disposition of drugs administered into the human Nasal cavity. Pharm. Res. 7 (1990) 69-75.

Gurny, R., Meyer, J.-M. and Peppas, N.A. Bioadhesive intraoral release systems: design, testing and analysis. Biomaterials, 5 (1984) 336-340.

Gurny, R. and Peppas, N.A., Semisolid dosage froms as buccal bioadhesives. In Lenaerts, V. and Gurny, R. (Eds), Bioadhesive Drug Delivery Systems, CRC Press, FL, 1990, pp. 153-168.

Hardy, J.D., Lee, S.W., and Wilson, C.G., Intranasal drug delivery by spray and drops. J. Pharm. Pharmacol, 37 (1985) 294-297.

Hunt, G., Kearney, P. and Kellaway, I.W., Mucoadhesive polymers in drug delivery systems. In Johnson, P. and Lloyd-Jones, J.G. (Eds), Drug Delivery Systems: Fundamentals and Techniques, Ellis Horwood, U.K., 1987, pp. 181-199.

Illum, L., Microspheres as a potential controlled release nasal drug delivery system. In Davis, S.S. (Ed.), Delivery Systems for Peptide Drugs, Plenum, New York, 1986, pp 205-210.

Leung, S.-H.S. and Robinson, J.R., Polymer structure features contributing to mucoadhesion: II. J. Controlled Release 12 (1990) 187-194.

Majima, Y., Hirata, K., Takeuchi, K., Hattori, M. and Sakakura, Y., Effects of orally administered drugs on dynamic viscoelasticity of human nasal mucus. Am. Rev. Respir. Dis., 141 (1990) 79-83.

Peppas, N.A., Hydrogels in Medicine and Pharmacy, Vol. II: Polymers, CRC Press, FL, 1987, pp. 115-160.

Peppas, N.A., Ponchel, G. and Duchene, D., Bioadhesive analysis of controlled-release systems: II. Time-dependent bioadhesive stress in poly(acrylic acid)-containing systems. J. Controlled Release, 5 (1987) 143-149.

Phillips, P.P., McCaffrey, T.V. and Kern, E.B., Measurement of human nasal ciliary motility using computerized microphotometry. Otolaryngol. Head Neck Surg., 103 (1990) 420-426.

Ponchel, G., Touchard, F., Duchene, D. and Peppas, N.A., Bioadhesive analysis of controlled-release systems: I. Fracture and interpenetration analysis in poly(acrylic acid)containing systems. J. Controlled Release, 5 (1987) 129-141.

Rajagopalan, N., Dicken, C.M., Ravin, L.J. and Sterson, L.A., A study of the solubility of amphotericin $B$ in nonaqueous solvent systems. J. Parenter. Sci. Technol., 42 (1988) 97-102.

Rubino, J.T. and Yalkowsky, S.H., Cosolvency and cosolvent polarity. Pharm. Res., 4 (1987) 220-230. 